\title{
Anisotropic convection in rotating proto-neutron stars
}

\author{
J. A. Miralles ${ }^{1}$, J. A. Pons ${ }^{1}$, and V. Urpin ${ }^{1,2}$ \\ ${ }^{1}$ Departament de Física Aplicada, Universitat d'Alacant, Ap. Correus 99, 03080 Alacant, Spain \\ 2 A. F. Ioffe Institute of Physics and Technology and Isaak Newton Institute of Chili, Branch in St. Petersburg, \\ 194021 St. Petersburg, Russia
}

Received 11 December 2003 / Accepted 17 February 2004

\begin{abstract}
We study the conditions for convective instability in rotating, non-magnetic proto-neutron stars. The criteria that determine stability of nascent neutron stars are analogous to the Solberg-Høiland conditions but including the presence of lepton gradients. Our results show that, for standard angular velocity profiles, convectively unstable modes with wave-vectors parallel to the rotation axis are suppressed by a stable angular momentum profile, while unstable modes with wave-vectors perpendicular to the axis remain unaltered. Since the wave-vector is perpendicular to the velocity perturbation, the directional selection of the unstable modes may result in fluid motions along the direction of the rotation axis. This occurs in rigidly rotating stars as well as in the inner core of differentially rotating stars. Our results provide a natural source of asymmetry for proto-neutron stars with the only requirement that angular velocities be of the order of the convective characteristic frequency.
\end{abstract}

Key words. stars: neutron - stars: rotation - stars: supernovae: general - hydrodynamics

\section{Introduction}

Hydrodynamical instabilities in newly born neutron stars play an important role in enhancing neutrino luminosities and increasing the energy deposition efficiency. It was first argued by Epstein (1979) that the negative lepton gradient that arises in the outer layers of the proto-neutron star (PNS) can be convectively unstable. However, convection in PNSs can be driven not only by the lepton gradient but by a negative entropy gradient as well (see, e.g., Arnett 1987; Burrows \& Lattimer 1986). The development of negative entropy and lepton gradients is common in many simulations of supernova models (Bruenn \& Mezzacappa 1994; Bruenn et al. 1995) and evolutionary models of PNSs (Burrows \& Lattimer 1986; Keil \& Janka 1995; Sumiyoshi et al. 1995; Pons et al. 1999). The nature of instabilities in PNSs has been considered by a number of authors (Grossman et al. 1993; Bruenn \& Dineva 1996; Miralles et al. 2000, MPU in the following; Miralles et al. 2002). In non-rotating and non-magnetic PNSs, the criteria indicate the presence of two essentially different instabilities (MPU) with a convectively unstable region surrounded by a neutron-finger unstable region, the latter involving typically a larger portion of the stellar material. The unstable zones grow in size and move inward as the PNS deleptonizes (Keil et al. 1996), until neutrino diffusion progressively reduces the temperature and lepton gradients, and convective instabilities disappear, at most in 30-40 s. The growth time of convective and neutron finger instabilities are substantially different, as well as the efficiency

Send offprint requests to: J. A. Pons, e-mail: Jose.A.Pons@uv.es of turbulent transport in the convective and neutron-finger unstable zones.

Less is known about convection in rotating PNSs. The failure of detailed simulations in spherical symmetry to get a successful Supernova explosion has led different authors to argue that multidimensional effects, such as convection or rapid rotation can be the key to the problem (see e.g. Buras et al. 2003, and references therein). From theoretical modeling and simple analytic considerations it is commonly accepted that core collapse of a rotating progenitor leads to differential rotation of a newly born neutron star (Zwerger \& Müller 1997; Rampp et al. 1998; Liu 2002; Dimmelmeier et al. 2002; Müller et al. 2004), mainly due to conservation of the angular momentum during collapse. A recent study on evolutionary sequences of rotating PNSs (Villain et al. 2004) shows that the typical scale on which the angular velocity changes is in the range $\approx 5-10 \mathrm{~km}$. Granted that a PNSs is born rapidly rotating, when the angular velocity is of the order or larger than the Brunt-Väisälä or Ledoux frequencies, even relatively fast convective instabilities can be substantially modified, and convection can be constrained to the polar regions (Fryer \& Heger 2000). In this paper, we study this effect in the context of PNSs.

\section{The dispersion relation}

Consider a PNS rotating with angular velocity $\Omega=\Omega(s, z)$. In what follows we use cylindrical coordinates $(s, \varphi, z)$. The deleptonization and cooling timescales are assumed to be much longer than the growth time of instability, thus it can be treated in a quasi-stationary approximation. For the sake of 
simplicity, we consider axisymmetric short-wavelength perturbations with spatial and temporal dependence $\exp (\gamma t-i \boldsymbol{k} \cdot \boldsymbol{r})$ where $\boldsymbol{k}=\left(k_{s}, 0, k_{z}\right)$ is the wave-vector. Small perturbations will be indicated by a subscript 1 , whilst unperturbed quantities will have no subscript, except when indicating vector components. In the unperturbed state, the PNS is in hydrostatic equilibrium,

$$
\frac{\nabla p}{\rho}=\boldsymbol{G}, \quad \boldsymbol{G}=\boldsymbol{g}+\Omega^{2} \boldsymbol{s}
$$

where $\boldsymbol{g}$ is the gravity.

In the Boussinesq approximation, the linearized momentum and continuity equations read

$\gamma \boldsymbol{v}_{1}+2 \boldsymbol{\Omega} \times \boldsymbol{v}_{1}+\boldsymbol{e}_{\varphi} s\left(\boldsymbol{v}_{1} \cdot \nabla \Omega\right)=\frac{i \boldsymbol{k} p_{1}}{\rho}+\boldsymbol{G} \frac{\rho_{1}}{\rho}$,

$\boldsymbol{k} \cdot \boldsymbol{v}_{1}=0$

where $\boldsymbol{v}_{1}, p_{1}$ and $\rho_{1}$ are perturbations of the velocity, pressure and density, respectively; $\boldsymbol{e}_{\varphi}$ is the unit vector in the azimuthal direction. We assume that the matter is in chemical equilibrium, thus the density is generally a function of the pressure $p$, temperature $T$ and lepton fraction $Y$. In the Boussinesq approximation, perturbations of the pressure are small, therefore $\rho_{1}$ can be expressed in terms of the perturbations of temperature, $T_{1}$, and lepton fraction, $Y_{1}$,

$\rho_{1} \approx-\rho\left(\beta \frac{T_{1}}{T}+\delta Y_{1}\right)$,

where $\beta$ and $\delta$ are the coefficients of thermal and chemical expansion; $\beta=-(\partial \ln \rho / \partial \ln T)_{p Y}, \delta=-(\partial \ln \rho / \partial Y)_{p T}$.

We consider instabilities arising on a timescale much shorter than the dissipative timescale, i.e., those perturbations with relatively long wavelengths but still smaller than the pressure scale, to ensure that our approximation is valid. For example, perturbations with $\lambda=2 \pi / k \sim 0.3-1 \mathrm{~km}$ satisfy both these requirements. For such perturbations, we can neglect dissipative effects in the equations governing thermal balance and lepton fraction (see MPU for more details about the influence of dissipative terms). Then, the linearized transport equations read

$$
\begin{aligned}
& \dot{T}_{1}-\boldsymbol{v}_{1} \cdot \Delta \nabla T=0, \\
& \dot{Y}_{1}+\boldsymbol{v}_{1} \cdot \nabla Y=0,
\end{aligned}
$$

where $\Delta \nabla T$ is the super-adiabatic temperature gradient:

$$
\Delta \nabla T=\left(\frac{\partial T}{\partial p}\right)_{s, Y} \nabla p-\nabla T
$$

The dispersion equation corresponding to Eqs. (1)-(6) is

$\gamma^{2}\left(\gamma^{2}+q^{2}-\omega_{g}^{2}-\omega_{L}^{2}\right)=0$

where

$q^{2}=\frac{k_{z}^{2}}{k^{2}} \Omega_{\mathrm{e}}^{2}-\frac{k_{s} k_{z}}{k^{2}} \Omega_{z}^{2}$,

$\omega_{g}^{2}=-(\beta / T) \boldsymbol{G}_{\perp} \cdot \Delta \nabla T, \quad \omega_{L}^{2}=\delta \boldsymbol{G}_{\perp} \cdot \nabla Y$, and $\boldsymbol{G}_{\perp}=\boldsymbol{G}-\boldsymbol{k}(\boldsymbol{k} \cdot \boldsymbol{G}) / k^{2} ; \Omega_{\mathrm{e}}$ is the epicyclic frequency, $\Omega_{\mathrm{e}}^{2}=\frac{1}{s^{3}} \partial\left(s^{4} \Omega^{2}\right) / \partial s$, and we have defined $\Omega_{z}^{2}=s \partial \Omega^{2} / \partial z$. Equation (7) differs from the standard dispersion equation of buoyant waves in a differentially rotating fluid (see, e.g., Goldreich \& Schubert 1967) by the presence of a term proportional to $\nabla Y$.

Non-trivial solutions of Eq. (7) are given by

$\gamma^{2}=-q^{2}+\omega_{g}^{2}+\omega_{L}^{2}$

The condition of instability is $\omega_{g}^{2}+\omega_{L}^{2}>q^{2}$. In non-rotating PNSs, the characteristic frequencies in the convective regions are typically of the order of $1 \mathrm{~ms}^{-1}$ (MPU). Therefore, rotational effects will be important when the angular velocity $\Omega$ is of the same order, $\Omega \approx 1000 \mathrm{rad} / \mathrm{s}$, which can be reached during the early stages of PNS evolution (Villain et al. 2004).

\section{The instability criteria.}

Assume that a vector $\boldsymbol{k}$ forms an angle $\phi$ with the polar axis. Defining the following vector,

$C=-(\beta / T) \Delta \nabla T+\delta \nabla Y$,

the dispersion relation can be written in terms of the components of $\boldsymbol{C}$ and $\boldsymbol{G}$ as follows:

$\gamma^{2}=A \cos ^{2} \phi+B \sin \phi \cos \phi+C_{z} G_{z}$

where $A=-\Omega_{\mathrm{e}}^{2}+C_{s} G_{s}-C_{z} G_{z}$ and $B=\Omega_{z}^{2}-C_{z} G_{s}-C_{s} G_{z}$. By taking the curl of Eq. (1), it can be readily obtained that the condition of hydrostatic equilibrium leads to

$\Omega_{z}^{2}=[\boldsymbol{C} \times \boldsymbol{G}]_{\varphi}=C_{z} G_{s}-C_{s} G_{z}$

so that $B$ can be further simplified to obtain $B=-2 C_{s} G_{z}$.

Since the dependence of Eq. (10) on $\phi$ is rather simple, we can obtain that the maximum of $\gamma^{2}$ corresponds to

$\cos ^{2} \phi=\frac{1}{2}\left[1 \pm \sqrt{\frac{A^{2}}{A^{2}+B^{2}}}\right]$,

with the \pm sign depending on the sign of $A$, and the maximum value of $\gamma^{2}$ is given by

$$
\begin{aligned}
\gamma_{\max }^{2}= & \frac{-\Omega_{\mathrm{e}}^{2}-\omega_{0}^{2}}{2} \\
& +\frac{1}{2} \sqrt{\left[\Omega_{\mathrm{e}}^{2}+\omega_{0}^{2}\right]^{2}+4 G_{z}\left(C_{z} \Omega_{\mathrm{e}}^{2}-C_{s} \Omega_{z}^{2}\right)} .
\end{aligned}
$$

where $\omega_{0}^{2}=-(\boldsymbol{C} \cdot \boldsymbol{G})$. The two conditions for stability follow straightforwardly from the above expression:

$-\Omega_{\mathrm{e}}^{2}-\omega_{0}^{2}<0 ; \quad G_{z}\left(C_{z} \Omega_{\mathrm{e}}^{2}-C_{s} \Omega_{z}^{2}\right)<0$.

These two conditions look like the Solberg-Høiland conditions (Tassoul 2000), but with additional terms due to the lepton gradients. Criteria (13) can be also applied to quasi-Keplerian toroidal configurations made of nuclear matter surrounding black holes, in which the effect of lepton gradients is relevant.

Notice that if isobaric and isopicnic surfaces coincide, $C$ and $\boldsymbol{G}$ are aligned, and vertical gradients of $\Omega$ are not allowed. 


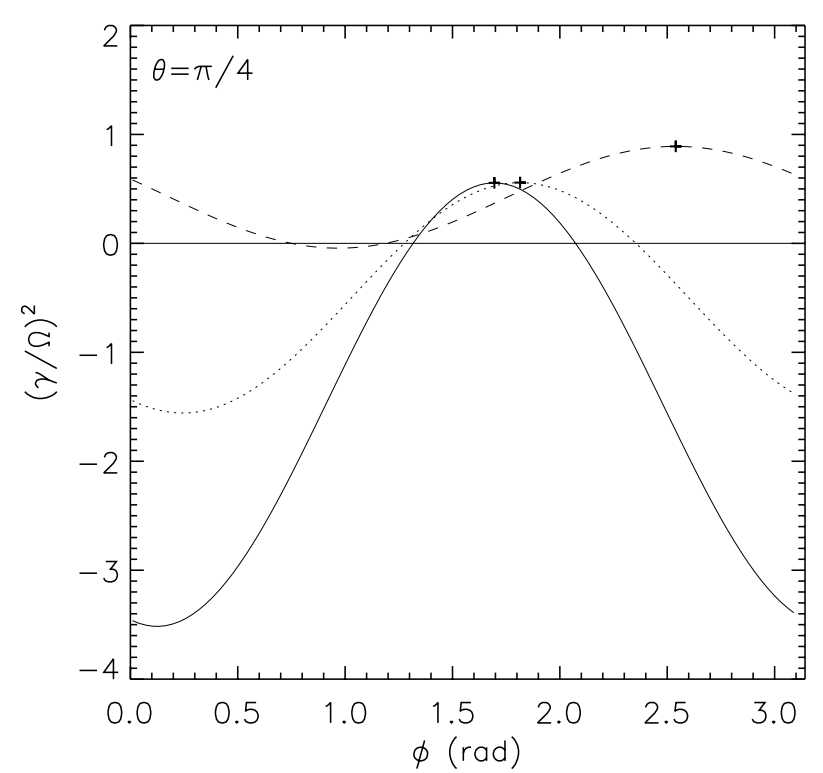

Fig. 1. Characteristic squared frequency normalized to the rotation frequency, $\gamma^{2} / \Omega^{2}$, as a function of the angle between the wavevector and the polar axis $(\phi)$, assuming that $\omega_{0}^{2}=\Omega^{2}$. The rotation law is described by Eq. (14). Three different radial points, with $s / R_{0}=0.1,1$, and 5 are plotted with solid lines, dots, and dashes, respectively.

Consistently, most of the studies of rotating stars with simplified polytropic equations of state use angular rotation profiles that depend only on $s$. In our calculations, we use the following law proposed by Komatsu et al. (1989) and used by many authors:

$\Omega(s)=\frac{\Omega_{c} R_{0}^{2}}{s^{2}+R_{0}^{2}}$.

In Fig. 1 we show $\gamma^{2} / \Omega^{2}$ as a function of the wavevector orientation in a convectively unstable situation, $\omega_{0}^{2}>0$. We have taken $\omega_{0}^{2}=\Omega^{2}$, and the polar angle $\theta=\pi / 4$. For simplicity, we have used the law (14) for the angular velocity so that $\Omega_{z}=0$. Three different cases are plotted with solid lines, dots, and dashes, corresponding to $s / R_{0}=0.1,2$, and 5 , respectively. When $s / R_{0} \ll 1$ the angular velocity varies slowly and the situation is close to rigid rotation, while for $s / R_{0} \gg 1$ the specific angular momentum is nearly constant and rotational effects are negligible. When the angular momentum increases with the distance to the axis (Rayleigh stable), $\gamma^{2}$ can become negative and instability is suppressed for a subset of the possible orientations of $\boldsymbol{k}$. Only perturbations with $\boldsymbol{k}$ oriented more or less perpendicular to the polar axis (fluid motions in the vertical direction) are not suppressed. Note that the fact that rotation results in turbulent convective motions preferentially in the direction parallel to the rotation axis was first pointed out by Randers (1942) in the context of the study of energy transport in stellar convective cores.

This effect is more manifest looking at Figs. 2 and 3. In Fig. 2 we plot the maximum value of $\gamma^{2}$ as a function of the polar angle $(\theta)$, at three different spherical radii. Near the pole the maximum of $\gamma$ does not change, but as we approach the equator, rotational effects may reduce its value drastically, unless the gradient of the angular momentum is small

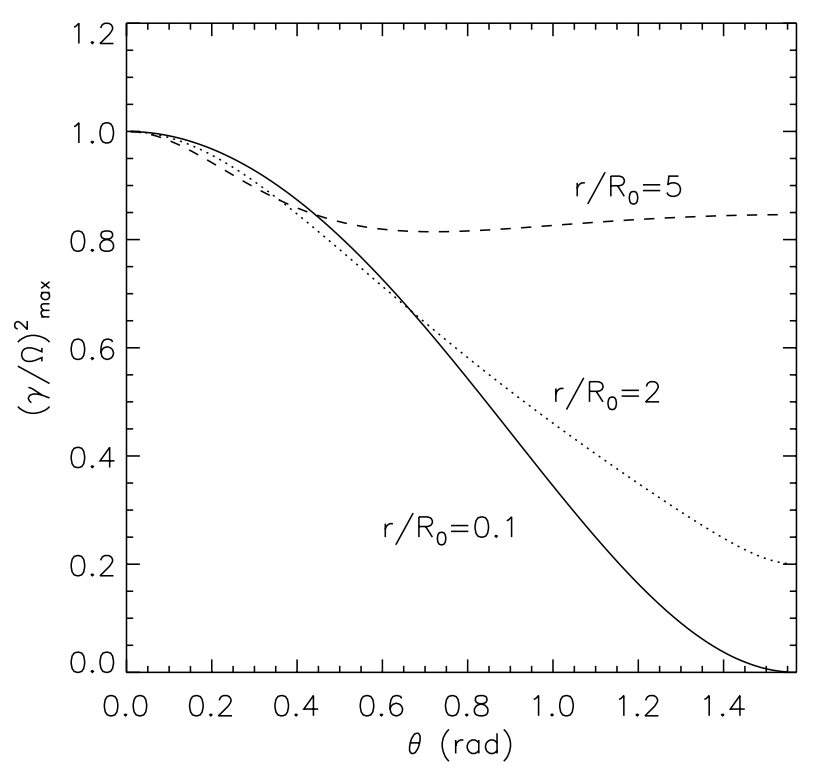

Fig. 2. Maximum of $\gamma^{2} / \Omega^{2}$ as a function of the polar angle for a fixed radius of $r / R_{0}=0.1$ (solid), 2.0 (dots) and 5.0 (dashes).

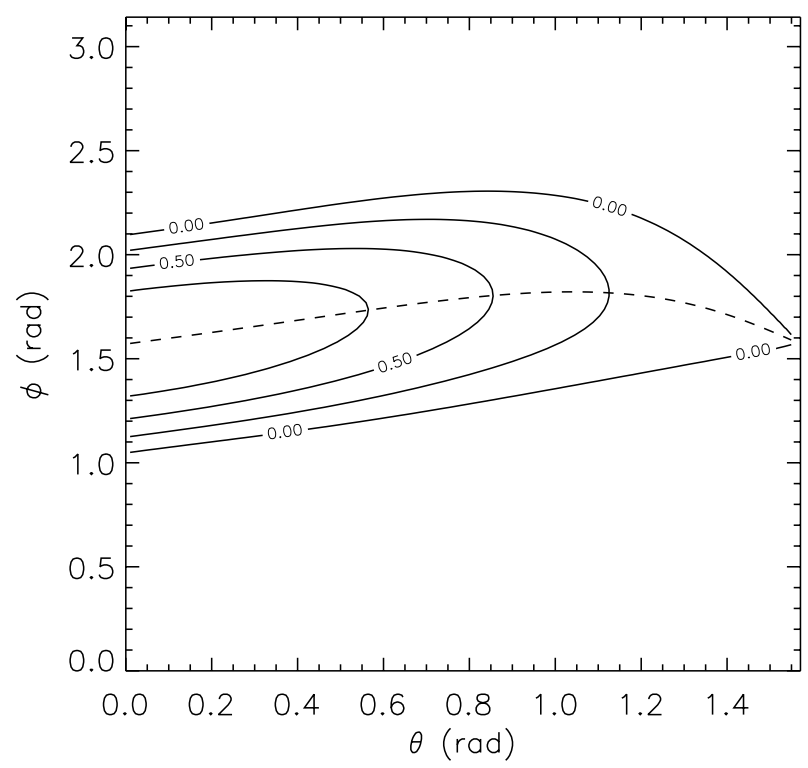

Fig. 3. Surfaces of constant $\gamma^{2} / \Omega^{2}$ in a $\theta-\phi$ plane, for $r / R_{0}=1$. The region enclosed by the surface $\gamma^{2} / \Omega^{2}=0$ is convectively unstable. The dashed line indicates the angle at which the maximum is reached.

(case $r / R_{0}=5$ ). More importantly, not only does the growth time become longer, but the unstable perturbations are restricted to some range of angles, centered at the value given by Eq. (12), and width given by

$\tan \Delta \phi=\frac{\sqrt{-G_{z}\left(C_{z} \Omega_{\mathrm{e}}^{2}-C_{s} \Omega_{z}^{2}\right)}}{\left(-\Omega_{\mathrm{e}}^{2}-\omega_{0}^{2}\right)}$.

In Fig. 3 we plot surfaces of constant $\gamma^{2} / \Omega^{2}$ in a $\theta-\phi$ plane, for $r / R_{0}=1$. The curve $\gamma^{2}=0$ encloses the interval of angles in which perturbations are unstable. The position of the maximum is indicated by the dashed line. Notice that when the second of the stability conditions (13) is not fulfilled, 
the function $\gamma^{2}$ has no zeros. Therefore, $\gamma^{2}$ is either positive or negative for all angles depending on the sign of the first condition.

\section{Discussion}

We have derived the criteria for convective instability in rotating, non-magnetic PNSs, obtaining two conditions analogous to the Solberg-Høiland criteria but including the effects of lepton gradients. In convectively unstable regions, the existence of a positive gradient of angular momentum can stabilize the unstable convective modes in some directions. The basic picture is that an unstable buoyant element of fluid is stabilized when it tries to move toward a direction in which the surrounding fluid elements have larger angular momentum, because it is subject to a smaller centrifugal force and therefore restored to its equilibrium position. However, this restoring force has no effect on buoyant waves that follow lines of constant angular momentum. For example, in a situation in which thermal, lepton, and pressure gradients are nearly aligned, the angular momentum depends weakly on the vertical coordinate, thus allowing convectively unstable modes to grow only in the direction approximately parallel to the rotation axis. In a general case, the geometry will be more complicated but the qualitative results will not change. In Fig. 4 we illustrate this effect in a realistic model of a PNS $0.5 \mathrm{~s}$ after birth, taken from Pons et al. (1999). The convectively unstable region extends from 17 to $28 \mathrm{~km}$, and the arrows indicate the direction of the most rapidly growing mode, with the length being proportional to the inverse growth time. In the convectively unstable region near the equator, $\Omega_{\mathrm{e}}$ varies between 500 and $1000 \mathrm{rad} / \mathrm{s}$, which is about one third the value of $\omega_{0}$, and we have taken $R_{0}=10 \mathrm{~km}$ (defined in Eq. (14)). In a saturated state, hydrodynamical non-linear effects will probably smear out anisotropies on small scales but it is unlikely that this may occur on the largest convective scale. Hydrodynamic simulations of the early evolution of rotating PNSs show that the scenario drawn in Fig. 4 is fulfilled during the very early phase of convection in PNSs (Janka, private communication), which shows that our linear stability analysis reflects accurately the situation at the onset of convection. However, after several hundreds of milliseconds, the efficient neutrino and convective transport in the polar region will flatten the temperature, lepton and angular momentum profiles, resulting in a different situation: convection occurring only close to the equator, although convective shells are still elongated and parallel to the rotation axis (see e.g. Fig. 10 in Janka \& Keil 1997; or Janka et al. 1997).

This coupling between convective instability and stabilizing gradients of angular momentum can be another ingredient that helps to explain the large asymmetry that has been observed in recent simulations of rotational core collapse Supernovae (Müller et al. 2003), not observed in simulations of convective PNSs without rotation. The mechanism we propose is a natural way to create anisotropic energy and momentum transport by convective motions, that only requires that the angular velocity be of the order of the Brunt-Väisälä or leptonic frequencies. Notice that our results are relevant for convective motions in the PNS, more than in the extended envelope as in Fryer \& Heger (2000). Our analysis is only valid

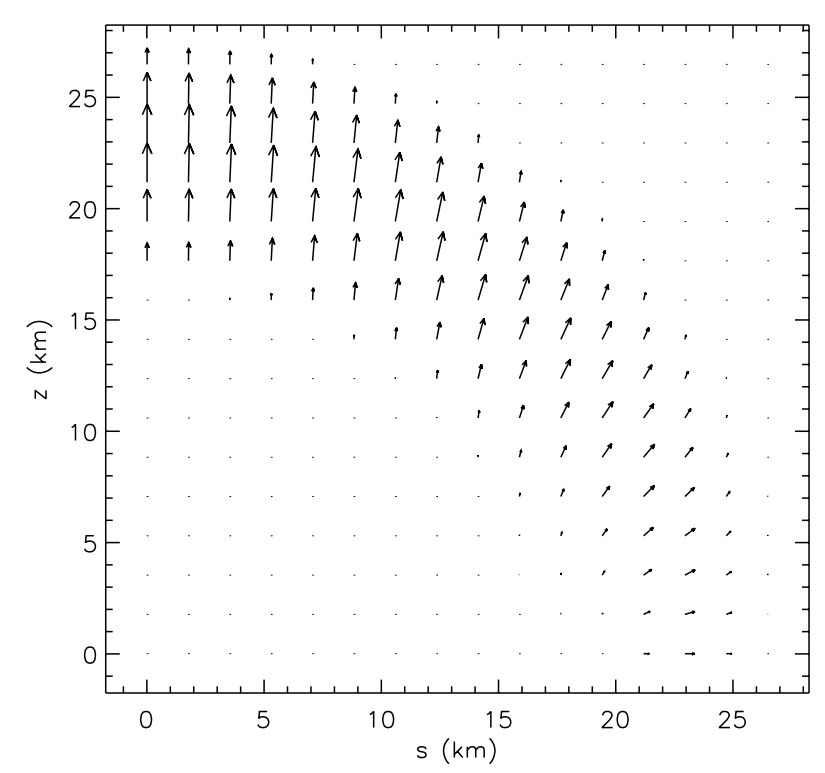

Fig. 4. The direction of the motion for the most rapidly growing modes in a realistic model of PNS $0.5 \mathrm{~s}$ after birth. In the convective region $(17-28 \mathrm{~km})$, the instability is more effective in the polar region and, even at intermediate latitudes, vertical motions are preferred.

in a steady-state situation, while the conditions in the accretion layer are extremely non-stationary, and other effects such as anisotropic accretion are certainly more relevant. If the collapsed core shows strong differential rotation, at scales larger than $50-100 \mathrm{~km}$ the angular momentum can be nearly constant and rotational effects are small. Nevertheless, the anisotropy originated in the inner core may have some effect at larger scales, and can help to explain the Supernova mechanism. Obviously, self-consistent 3D simulations involving convection, neutrino transport and angular momentum transport are needed prior to drawing robust conclusions.

In our study we only considered non-magnetic protoneutron stars. The presence of a large magnetic field could change this picture. It has recently been shown that turbulent mean-field dynamo can be effective for PNSs with periods shorter than $1 \mathrm{~s}$, which would generate strong magnetic fields in the interior (Bonanno et al. 2003). However, magnetic field effects begin to be relevant only when the Alfvén frequency is of the order of the other dynamical or dissipative frequencies, and this requires magnetic fields larger than $10^{15} \mathrm{G}$ (Miralles et al. 2002). Probably the missing ingredient to explain Supernovae is related to the coupling between different effects, such as the one proposed in this paper, the magneto-rotational instability (Akiyama et al. 2003), or a combination of all of them.

Acknowledgements. This work has been supported by the Spanish Ministerio de Ciencia y Tecnología grant AYA 2001-3490-C02. J.A.P. is supported by a Ramón y Cajal contract from the Spanish MCyT. V.U. is supported by the Russian Foundation of Basic Research (04-02-16243) and a grant from Generalitat Valenciana. We thank the referee of this paper (H.-Th. Janka) for useful comments and sharing unpublished results with us. 


\section{References}

Akiyama, S., Wheeler, J. C., Meier, D., \& Lichtenstadt, I. 2003, ApJ, 584,954

Arnett, W. D. 1987, ApJ, 319, 136

Bonanno, A., Rezzolla, L., \& Urpin, V. 2003, A\&A, 410, L33

Bruenn, S., \& Dineva, T. 1996, ApJ, 458, L71

Bruenn, S., \& Mezzacappa, A. 1994, ApJ, 433, L45

Bruenn, S., Mezzacappa, A., \& Dineva, T. 1995, Phys. Rep., 256, 69

Buras, R., Rampp, M., Janka, H.-Th., \& Kifonidis, K. 2003, Phys. Rev. Lett., 90, 1101

Burrows, A., \& Lattimer, J. M. 1986, ApJ, 307, 178

Dimmelmeier, H., Font, J. A., \& Müller, E. 2002, A\&A, 393, 523

Epstein, R. 1979, MNRAS, 188, 305

Fryer, C. L., \& Heger, A. 2000, ApJ, 541, 1033

Goldreich, P., \& Schubert, G. 1967, ApJ, 150, 571

Grossman, S. A., Narayan, R., \& Arnett, D. 1993, ApJ, 407, 284

Janka, H.-Th., \& Keil, W. 1997, in Supernovae and Cosmology, Proc. of the Colloquium in Honor of Prof. G. Tammann, Augst, Switzerland, June 13 [arXiv: astro-ph/9709012]
Janka, H.-Th., Kifonidis, K., \& Rampp, M. 2001, Lect. Notes Phys., 578,333

Keil, W., \& Janka, H.-Th. 1995, A\&A, 296, 145

Keil, W., Janka, H.-T., \& Muller, E. 1996, ApJ, 473, L111

Komatsu, H., Eriguchi, Y., \& Hachisu, I. 1989, MNRAS, 239, 153

Liu, Y. 2002, Phys. Rev. D, 65, 14003

Miralles, J. A., Pons, J. A., \& Urpin, V. 2000, ApJ, 543, 1001

Miralles, J. A., Pons, J. A., \& Urpin, V. 2002, ApJ, 574, 356

Müller, E., Rampp, M., Buras, R., Janka, H.-T., \& Shoemaker, D. 2004, ApJ, 603, 221

Pons, J. A., Reddy, S., Prakash, M., Lattimer, J., \& Miralles, J. A. 1999, ApJ, 513, 780

Randers, G. 1942, ApJ, 95, 454

Rampp, M., Müller, E., \& Ruffert, M. 1998, A\&A, 332, 969

Sumiyoshi, K., Suzuki, H., \& Toki, H. 1995, A\&A, 303, 475

Tassoul, J.-L. 2000, in Stellar Rotation (Cambridge: Cambridge Univ. Press)

Villain, L., Pons, J. A., Cerdá-Durán, P., \& Gourgoulhon, E. 2004, A\&A, 418, 283

Zwerger, T., \& Müller, E. 1997, A\&A, 320, 209 